\title{
Germanica
}

\section{Die selektive Aktualität des Hörspiels}

Le rapport sélectif de la pièce radiophonique à l'actualité

\section{Ute Eckelkamp}

\section{OpenEdition}

Journals

Édition électronique

URL : http://journals.openedition.org/germanica/2217

DOI : 10.4000/germanica.2217

ISSN : 2107-0784

Éditeur

Université de Lille

\section{Édition imprimée}

Date de publication : 1 décembre 1994

Pagination : 107-121

ISSN : 0984-2632

Référence électronique

Ute Eckelkamp, «Die selektive Aktualität des Hörspiels », Germanica [Online], 14 | 1994, Online erschienen am: 20 Januar 2014, abgerufen am 06 Oktober 2020. URL : http://

journals.openedition.org/germanica/2217 ; DOI : https://doi.org/10.4000/germanica.2217

Ce document a été généré automatiquement le 6 octobre 2020.

(c) Tous droits réservés 


\title{
Die selektive Aktualität des Hörspiels
}

\author{
Le rapport sélectif de la pièce radiophonique à l'actualité
}

\author{
Ute Eckelkamp
}

1 Das Hörspiel ist in erster Linie ein Genre des Mediums Rundfunk ${ }^{1}$, in dessen Rahmen es in seiner Beziehung zur Aktualität auch im Hinblick auf die anderen Gattungen betrachtet werden muß, die ihrerseits ein klar definiertes und zum Teil enges Verhältnis zum unmittelbaren Zeitgeschehen haben.

2 Aktualität im publizistischen Sinne ist zu verstehen als Neuigkeitswert einer Nachricht oder einer journalistischen Botschaft im Spannungsfeld von objektivem Bedeutungsgrad eines Ereignisses und dem subjektiven Intensitätsgrad der Erwartung.

3 Die Publizistik unterscheidet dabei zwischen primärer, akuter und sekundärer, latenter Aktualität ${ }^{2}$. Das Genre der primären Aktualität, die Nachrichten, so wie die daran anschließenden, vertiefenden Sendeformen schließen eine Auseinandersetzung des Hörspiels mit Aktualität nicht aus, zumal prinzipiell die Möglichkeit besteht, ein Hörspiel in unmittelbarer zeitlicher Nähe zum Geschehen selbst zu realisieren.

4 Per definitionem hat das Hörspiel den gleichen Programmauftrag wie alle anderen Rundfunkgattungen, nämlich Information, Bildung und Unterhaltung zu liefern. Diese Funktionen sind nicht an die Großgruppen des Programms gebunden, sondern gelten für jede Sendung, so daß das Hörspiel theoretisch durchaus in Konkurrenz zu den anderen Gattungen treten kann.

5 Helmut Heißenbüttel weist darauf hin, daß das Hörspiel sein Entstehen einer Auftragssituation verdankt ${ }^{3}$. Seine Funktion war $\mathrm{zu}$ Beginn die Füllung von Programmplatz, da die vorrangige Aufgabe des Funks die Vermittlung aktueller Information durch die Nachrichten war, während alle anderen Sendeformen nur Füllsel bildeten. Und auch hier rangierten die Spezialinteressen, die etwa in Land-, Kirchenoder Frauenfunk behandelt werden, vor den sogenannten kulturellen Programmen, zu denen das Hörspiel zählt. 
6 An den Programmplatz sind entsprechende Erwartung seitens des Hörers geknüpft, die Hellmut Geißner ${ }^{4}$ je nach Art ihres «Spiel-Waren-Charakters» dem ernsten oder Unterhaltungsbereich zuweist, wobei einige Sender das kulturelle Hörspiel den Hauptabteilungen Kultur und das Unterhaltungshörspiel (Volksstück, Mundarthörspiel, Krimi, science fiction) redaktionell der Unterhaltung zuweisen.

7 Analog $\mathrm{zu}$ den anderen Sendeformen kann das aktualitätsbezogene Hörspiel auf Realität reagieren. Im Unterschied $\mathrm{zu}$ diesen ist es in seiner Form jedoch nicht standardisiert, während Realität als Referenzgegenstand bedingt ist durch die Wahrnehmung des Beobachters. Während die unmittelbar auf Aktualität bezogenen Sendeformen wie Nachrichten und Reportage sowie sekundäre Formen wie Interview, Diskussion, Magazin und Feature geprägt sind durch die subjektive Realität von Kulturkreis, sozialer Gruppe und Individualität des jeweiligen Berichterstatters, die im Rahmen konventionalisierter journalistischer Formen eine «Medienrealität» schaffen ${ }^{5}$, ist das Hörspiel frei von solchen Standardisierungszwängen, und darin liegen seine unbegrenzten ästhetischen Möglichkeiten. In der Bezugnahme auf aktuelles Zeitgeschehen gibt es dabei zwei Möglichkeiten: nämlich erstens die unmittelbare Bezugnahme zum Geschehen, die den gleichen subjektiven Beschränkungen in der Wahrnehmung von Realität schlechthin unterworfen ist wie die des Journalisten, sowie bei beiden die an die akustischen Möglichkeiten des Mediums gebundene Wiedergabe der Wirklichkeit sowie zweitens die Bezugnahme des Hörspiels auf eben jene Medienrealität der anderen Sendeformen und weiterer Medien.

Medienrealität ist ihrerseits schon Resultat eines selektiven Vorgangs entsprechend den Kategorien Arnolds und Verres. Das Hörspiel in seiner Auseinandersetzung mit Aktualität steht also entweder neben diesen standardisierten Rundfunkformen, und zwar in - kritischer - Anlehnung an diese, indem es sie als Quelle nutzt oder gar als Gegenstand der Auseinandersetzung verwendet. Aktualität, die in eben dem Medium geschaffen wird, das als ihr Multiplikator fungiert, wird durch die Reflexion dieses Mediums selbst und seines Umgangs mit dem jeweiligen Stoff zum zweiten Thema des aktuellen Hörspiels, das Medium selbst zum ersten, wobei die Gewichtung zwischen Zeitgeschehen und Medienthematik nicht unbedingt Prioritäten setzen muß, wie die anschließenden Beispiele zeigen.

9 Dies schließt eine Bezugnahme auf die jeweiligen Rundfunkformen ein, zu denen das Hörspiel dabei in eine gefährliche Nähe rücken kann, denn indem es sich - unter Verwendung realer oder fiktionaler Stoffe - deren Sprache und Zeichen bedient, ist die Unterscheidung zwischen Hörspiel und anderen Formen in bestimmten Fällen nur noch durch den Programmplatz, die An- und Absage und ev. durch bestimmte formale Merkmale der ästhetischen Gestaltung zu treffen. Daß auch dies nicht immer gewährleistet ist, zeigen Beispiele wie die CBS-Produktion «The War of the Worlds» von Orson Welles (1938) u.a.

Berührungspunkte und Ähnlichkeiten mit anderen Gattungen können vorkommen, oder sogar, wie im englischen Hörfunk beispielsweise unter dem Dach der Gattung «radio play», vereinigt sein. Hörspiel und Feature sind hier nicht in verschiedene Ressorts getrennt, und das Hörspiel, das sich mit aktuellem Zeitgeschehen beschäftigt, fiele somit unter die gleiche Definition wie das Feature:

The radio feature is a dramatised présentation of actuality but its author should be much more of a rapporteur or a cameraman; he must select his actuality material 
with great discrimination and then keep Control of it so that it subserves a single dramatic effect ${ }^{6}$.

Die zu erzielende Wirkung unterliegt einer vorher festgelegten Intention, die auf eine vorherige Interpretation des zeitgeschichtlichen Geschehens zurückgeht, während die anderen Genres sich um möglichst «objektive» und ausgewogene Berichterstattung bemühen. Im Hörspiel wird dagegen mittels der Intentionalität bereits ein erster Schritt in die Fiktionalität getan.

Dabei stehen dem Hörspiel eine Reihe von Typen der Verarbeitung zur Verfügung. In der Selektion seiner Themen und Stoffe kann es einen beliebigen zeitlichen Abstand bzw. beliebige Nähe zu den Fakten selbst wählen. Es hat somit die Möglichkeit, solche vor dem schnellen Vergessen zu bewahren oder aber sich Themen zu widmen, die in den anderen Sendeformen keinen oder $\mathrm{zu}$ wenig Platz erhalten, z.B. das Unspektakuläre und die alltägliche Realität. Gleichzeitig steht es dem Hörspiel offen, selbst Aktualität herzustellen in der Schaffung neuer Formen und Themen. Dabei hat das Hörspiel die Möglichkeit, Themen fiktional anzugehen, dokumentarisch nachzuspielen oder mit Original-Tönen zu arbeiten.

In folgenden sollen einige Beispiele für Hörspiele vorgestellt werden, die sich parallel zum zeitgeschichtlichen Thema zugleich mit der medienrealen Erscheinungsform dieses Geschehens beschäftigen. Dabei seien neben dem inszenierten, fiktionalen Stück, das sich eines journalistischen Genres bedient, wie z.B. Orson Welles in «The War of the Worlds», drei weitere Typen unterschieden: Ein nicht-fiktionales Thema (psychische Krankheit) an einem fiktionalen Beispiel (der Fall Monika) wird im Rahmen eines adäquaten Hörfunkgenres (fiktives Magazin «Der Fall von nebenan») dargestellt in «Gestörtes Leben» von Wolfgang Schiffer?

Zweites Beispiel ist die Gestaltung eines realen Ereignisses (Golfkrieg) in a) einer Mischung von O-Ton-Material mit gespielten Dialogen (Mayröcker), b) satirischem Kommentar mit Synchronisation (Federman) und c) fiktionalem Spiel (Jens) in «Wüstensturm» ${ }^{8}$.

Einen dritten Typus bildet das Originial-Ton-Hörspiel, in dem Ludwig Harig Funkaufzeichnungen eines realen Ereignisses (Adenauer-Begräbnis) zu einer Montage verarbeitet: «Staatsbegräbnis»" .

Große Nähe zum aktuellen Zeitgeschehen führt zu zweierlei Reaktionen: zum einen zur Verwechselung - man denke an die Massenpanik, die durch die Welles-Inszenierung entstand und erheblichen Sachschaden nach sich zog. Verwechselungen sollten auf Grund möglicher Schädigungen von Personen ausgeschlossen werden. Zum anderen steigert diese Nähe zweifellos die Attraktivität des Hörspiels, das im Grunde eine immer schmalere Hörerschaft zählt.

7 In der Bezugnahme auf Zeitgeschehen durch Anlehung an Formen anderer Sendungen besinnt das Hörspiel sich auf seine eigenen Bedingungen und nimmt gleichzeitig eine medienkritische Haltung ein. Hier findet ein weiterer selektiver Prozeß statt, der bewußt neben der Wahl des Sujets erfolgt und das Stück jeweils um eine Dimension erweitert.

Das Stück «Gestörtes Leben» von Wolfgang Schiffer wählt das Magazin als Rahmen, dem das Thema, der Umgang mit psychisch kranken Menschen als «Fall von nebenan» im Alltag als latenter Aktualität und der Behandlung dieses Stoffes in der Medienrealität vollkommen entspricht. Daß der Fall fiktional ist, bleibt nebensächlich. 
Es geht um eine junge Frau, die sich wieder in ein sogenanntes normales Leben einzugliedern versucht. Diese Eingliederung begleitet ein Journalist und Moderator. Während dieser Recherche gemachte Aufnahmen sind wie üblich in der MagazinSendung als eingespielte Beiträge zwischen Studio-Gesprächen mit anderen Informanten und Telefongesprächen im Studio zu hören. Die Integration der psychisch kranken Frau mißlingt jedoch u.a. dadurch, daß sie feststellen muß, daß der Journalist kein menschliches Interesse für sie empfindet, sondern sie zu einem «Fall» für eine Sendung funktionalisiert. Anders als im herkömmlichen Magazin werden die Musiktitel nicht vollständig ausgespielt und außerdem stellenweise eine "mise en abyme " per Sampler der Magazin-Ton in eine Radio-Atmosphäre versetzt und darüber die Stimme der Protagonistin gelegt, die die Sendung über ihren «Fall» am Gerät verfolgt und kommentiert. Im Unterschied zum realen Magazin thematisiert der Moderator sein Fehlverhalten während der Recherche in der eigenen Sendung. Diese Funktion als AntiHeld ist in einer realen Magazinsendung weder üblich noch angebracht.

so jedoch gelingt es in dem Stück, neben der aktuellen Problematik des psychischen Krankseins die Schwierigkeit des Involviertseins des Berichterstatters in seinen Gegenstand zu beschreiben und Kritik an der Funktionalisierung menschlicher Probleme für die Füllung von Sendezeit zu üben.

Eine simultane Reaktion auf aktuelles Geschehen ist das Rundfunkfresko «Wüstensturm». Die Hörspielredaktion des Bayerischen Rundfunks hatte sich hierfür an namhafte Hörspielautoren gewandt mit der Bitte, einen Beitrag zum Thema des Golfkriegs zu liefern. Die eingesandten Manuskripte von Friederike Mayröcker, Raymond Federman und Walter Jens wurden von Ulrich Gerhard realisiert. Die «offene Form» faßt dabei im Grunde drei einzelne Kurzhörspiele zusammen, deren Zusammenhalt durch das gemeinsame Thema gewährleistet ist.

21 Der erste Text stammt von Friederike Mayröcker und beschreibt, ohne sich ausdrücklich auf den Golfkrieg zu beziehen, modernes Kriegsgeschehen aus der Perspektive eines Beobachters, die durchaus diejenige eines amerikanischen Militärs oder eines Journalisten sein könnte.

Sprechfunkstimmen, Störgeräusche aus Sprechgeräten und Kontaktaufnahmen über technische Kommunikationsapparaturen charakterisieren Ereignislosigkeit und Distanz zum unmittelbaren Kriegsgeschehen. Der Hörer erlebt die hilflose Distanz des modernen live-Berichterstatters, der vor Ort ist und doch kein eigentliches Ereignis zu schildern hat. Spannung und verständständnisloses Warten teilen sich dabei unmittelbar mit.

Darüber liegen die sehr präsent klingenden Stimmen eines Sprechers und einer Sprecherin, die einzelne Eindrücke mitteilen: zum Beispiel die Metall-Halskrause für ein Halswirbeltrauma. Die Verletzung wird nicht als Kriegsverletzung geschildert, der Verband erscheint als dem Menschen aufgezwungenes Korsett, dem Verletzten nicht angemessen und daher nicht in der Lage zu heilen. Die Vorgänge des eigentlichen Geschehens entziehen sich der unmittelbaren Wahrnehmung; Entfremdung und Distanz werden evident. Die Sprachlosigkeit angesichts des Nicht-Zugänglichen ist nun - verglichen mit früheren Darstellungen von Kriegen - nicht mehr die des Entsetzens, sondern stummer Ausdruck der Distanz und Entfremdung durch Entrückung. Sprache wird lückenhaft oder Pseudoinformation, sie wird als Informationsmittel monopolisiert (z.B. bei Nachrichtensperren). Durch die sachliche Sparsamkeit der hier vorgeführten Eindrücke scheint der Text gewissermaßen verallgemeinert, wozu auch die Anspielung 
der Sprecher auf den Konflikt zwischen Kain und Abel beiträgt. Mit dem Sprung von der aktuellen auf die archaische Ebene mythisiert Mayröcker das Phänomen Krieg. Sie konstatiert die Wiederholung des Immer-Gleichen, doch liegt in dem Archetypus von Kain und Abel auch ein individualisierendes Moment, da diese Gestalten immerhin personifizierte Individuen sind. Die Feindschaft zwischen Brüdern soll an die Nähe der Menschen untereinander, an das Gemeinsame mahnen.

Raymond Federman legte einen satirischen Brief an Hörspielleiter Herbert Kapfer vor. Er schildert darin die Geschichte des Golfkriegs als großartiges Medienspektakel mit einem ungeheuren Erfolg in der amerikanischen Öffentlichkeit. Wörtlich vergleicht Federman die Berichterstattung mit der soapopera und beschreibt vor diesem Hintergrund die Strukturen der Medienberichterstattung und deren Präsentation diverser Fakten. Der Krieg war ein gutes Schauspiel, weil es um einen gerechten Krieg ging, in dem die Amerikaner als die Guten und die Iraker als die Bösen auftraten. Gerecht war er, denn die Guten blieben Sieger.

Der Sprecher Federmann spielt den satten, zufriedenen Amerikaner im hemmungslosen Selbstbewußtsein des konsumbewußten Zynikers. Dagegen stehen in gleicher Lautstärke die weibliche Stimme der Simultanubersetzerin (Sigrid van der Laden), und der Autoren-Text sich in voller Lautstärke, verteilt auf die beiden Lautsprecher, gegenüber, und der Hörer wird gleichsam «in die Zange» genommen. Durch die intensive beiderseitige Beschallung entsteht ein Effekt von Unmittelbarkeit und akustischer Dichte, die sich auf die Wahrnehmung der Inhalte überträgt und eine gewisse Aktualisierung bewirkt. Hörgewohnheiten in Bezug auf Simultanübersetzungen in Kommentaren zu aktuellen Themen werden hier zum Instrument eines neuen tieferen Verständnisses. Das eben erst Vergangene und in den Medien bereits Überholte wird so zurückgeholt in das Bewußtsein und das allgemeine Interesse. Hier sind es jedoch inoffizielle «insider»-Informationen, d.h. der Brief übermittelt so etwas wie inoffizielle Nachrichten, wozu die intime Briefform die Hand reicht. Hauptakteure sind nun die Menschen im amerikanischen Alltag: die Leute tragen die in Mode gekommenen Wüstensturm-Uniformen im Büro, da sie als modische Aktualität für schick gehalten werden, und unter dem Ladentisch werden die 50000 Leichensäcke verkauft, für die es keine Verwendung mehr gab, da man die Toten auf der eignen Seite zu zahlreich kalkuliert hatte.

Auch hier wird ein Seitenblick auf den Medieneffekt geworfen: Federman verweist in einer abschließenden Anekdote auf den isrealischen Dirigenten Sterne, der bei einer Warnung während eines Mozart-Konzerts als einziger darauf verzichtet, die Gasmaske aufzusetzen und nur ein Schulterzucken für die Situation übrig hat. Federman löst die Geste in zwei Bedeutungsmöglichkeiten auf: Ich werde sowieso irgendwann bald sterben müssen, oder: Die Show muß ja doch weitergehen.

Aktuelles Zeitgeschehen ist für die Medienöffentlichkeit zum Unterhaltungsprogramm geworden, zur sogenannten MegaShow, die Kriegsberichterstattung versteht sich als live-Spektakel, das nicht nur simultan verfügbar gemacht wird, sondern alle Lebensbereiche als umfassende Konsummöglichkeit erreicht.

Das Stück von Walter Jens erzählt von den «absent soldiers». Der Friedensinitiative «Gustav Heinemann» geht es darum, sich aktiv gegen den Golf krieg zu wenden. Gelegenheit dazu bietet sich, als desertierende Soldaten der US-Armee untergebracht, versteckt und beschützt werden müssen. Formal ist auch dieses Stück unspektakulär: 5 Stimmen aus der Friedensgruppe schildern ihre Aktionen und deren Erfolg, ihre 
Gefühle und Eindrücke, guten Willen, peinlichen Kleinmut und anschließende Scham. Nach einigen schamerfüllten Bedenken entschließt sich ein prominentes Ehepaar aus der Gruppe, zwei Soldaten aufzunehmen, einen Schwarzen und eine Frau. Berührungsängste gegenüber den potentiellen «underdogs» erweisen sich schließlich als unbegründet, doch am Ende des eher positiven Zusammenlebens steht die Verurteilung der «absent soldiers» zu 5 Jahren Haft und anschließender Perspektivlosigkeit und Diskriminierung, während die deutschen Helfer sich ohne große Risiken und Aufwände ein reines Gewissen verschafft haben.

Auch hier kommt durch den impliziten Vergleich mit den eigentlichen Betroffenen ein Stück Distanz zum Ausdruck. Unmittelbare existentielle Betroffenheit und deren Konsequenzen bei der Verweigerung gegenüber dem Kriegsgeschehen werden der institutionalisierten Solidarität in der einmal monatlich tagenden Friedensgruppe gegenübergestellt. Sobald es jedoch um konkrete Aktion und kleine Risiken geht, stellen sich gleich Bedenken und Widerstände ein, und durch einen Schritt vorwärts in wirkliches Involviertsein geht bei den Friedensgruppen-Mitgliedern ein winziger, aber elementarer Lernprozeß vor.

Jeder der drei Autoren wählt ein spezifisches Phänomen, mit dem Distanz vom eigentlichen Kriegsgeschehen zum Ausdruck kommt: Mayröcker zeigt die Distanz der unmittelbar Beteiligten vor Ort, denen das Geschehen und seine Ursachen unzugänglich bleiben und die lediglich die Möglichkeit haben, Bruchstücke einer sekundären Realität $\mathrm{zu}$ beobachten. Problematisiert wird nun weniger das Kriegsgeschehen selbst, Atavismus eines alten Konflikts, der immer wieder zwischen Menschen aufzubrechen droht, als die Entfremdung, bei der die Gegner in immer weitere Distanz zueinander geraten und der Kriegszustand abstrakt aber permanent zu werden droht.

31 Federman zeigt die Verarbeitung des Geschehens zum Medienspektakel, zum Konsumartikel, betrachtet durch die zynische Haltung eines Beobachters, der scheinbar auf Weltverbesserung verzichtet. Federman geht sogar so weit, mit dem Dirigentenzitat zu zeigen, daß selbst die Betroffenen ihr Involviertsein in Zeitgeschichte für irrelevant erklären und eine fatalistische Haltung einnehmen.

Die Distanz bei Jens entsteht durch die faktische Entfernung des Kriegsgeschehens von den Personen des Stückes, die im weit entfernten Deutschland durch eine Gewissensentscheidung sich der Sache annehmen und ihr Involviertsein wählen, aber dennoch unfähig sind, die eigentlichen Probleme zu lösen. Hier wird Hilflosigkeit auf allen Ebenen spürbar gemacht.

«Das Staatsbegräbnis oder Vier Lektionen politische Gemeinschaftskunde» wurde 1969 von Ludwig Harig in der Regie von Johann M. Kamps im Saarländischen Rundfunk produziert. Das Stück steht in der Reihe des Neuen Hörspiels, in der bereits Harigs «Blumenstück» von 1968 als Klassiker gilt. Für das «Staatsbegräbnis» verwendet er ausschließlich Originaltöne aus dem Funkarchiv, die von den Trauerfeiern für Konrad Adenauer stammen. Dieses Material war in der Bundesrepublik weithin bekannt, vergleichbar nur mit den Mondflug-Dokumenten, die Ferdinand Kriwett in «Apollo Amerika» zum Hörspiel verarbeitet hatte ${ }^{10}$.

Harigs Montage nimmt Bezug auf die Begräbnisfeiern, die nach dem Vorbild der Beisetzung Churchills organisiert waren ${ }^{11}$. 

Beschwörungscharakter, indem er das Pathos durch seine Wiederholung lächerlich macht. Die Grundsätze selbst werden durch übertriebene Dichte und Häufigkeit als Leerformeln kenntlich gemacht. Das O-Ton-Hörspiel fungiert hier als sogenanntes «Sprachdenkspiel», in dem über die implizite Kritik an der Sprache Ideologiekritik geübt wird. Ist die Aktualität durch den zweijährigen Abstand zum Ereignis selbst auch relativ, so sorgt der Bekanntheitsgrad des Materials doch für eine große Attraktivität, die in der Collage den Hörer zwingt, sich selbst, seine Hörgewohnheiten und seine Akzeptanz gegenüber den Massenmedien $\mathrm{zu}$ reflektieren. Die Hörerreaktionen bestätigten dies. Nach Bodo Wurffei wird dem Rezipienten seine eigene Unmündigkeit 
vorgeführt, indem der Autor die im experimentellen Hörspiel angelegte Gelegenheit nutzt, mit dem Medium selbst zu spielen ${ }^{12}$.

Das Massenmedium wird dabei kritisiert, weil es sich im Sinne des politischen Machthabers funktionalisieren läßt. Dazu stellt Harig am Anfang und Ende des Stückes stimmungsvolle Passagen der Reporter neben solche, in denen sie Kontakt zur Sendeleitung aufnehmen und den Inszenierungscharakter des Nachfolgenden und Vorhergegangenen preisgeben, also Elemente, die allenfalls auf Grund von Pannen ausgestrahlt werden. Dieser Blick hinter die Kulissen erzeugt einen weiteren Effekt der Desillusionierung.

In die entgegengesetzte Richtung zielt die Übertreibung der sakralen Aura. Sie kommt vor allem durch die Dominanz der religiösen Elemente - Musik, Raumatmosphäre, Zitate und Liturgie - zustande. Die Parodie durch die Collage geht dabei nur so weit, daß die Funktion dieser Versatzstücke deutlich, ihr Ernst aber gewahrt bleibt.

Zur Sakralisierung trägt auch die Tatsache bei, daß Adenauers Name ausgespart bleibt. Zwar war dies bei der Bekanntheit der Person und auch des Ton-Materials nicht nötig, doch die Aussparung bewirkt eine Verallgemeinerung, bei der Adenauer nur noch als Beispiel für das Funktionieren staatlicher Macht dient. Außerdem erfolgt durch die Anonymität in Anbindung an die sakrale Aura eine Überhöhung der angedeuteten Person, die sie in die Position einer höheren, metaphysischen Macht rückt. Diese Stellung wird durch die Attribute der Person gestützt, die, in den Kontext von Liturgie und sakralem Diskurs eingebettet, untereinander in neue Bezüge gestellt werden.

Die Mechanismen bürgerlicher Verehrung geraten so in den Verdacht irrationaler Anbetung, inszeniert von Politikern, Kirche und Massenmedien. Die Trauerfeier wird zur Anbetung personifizierter Staatsmacht, die in dieser Art Gottesdienst an die Stelle der metaphysischen Macht rückt.

Die Beschwörung von Größe erfolgt als Synedoche «der große Tote», «der große alte Mann» ${ }^{13}$, als Größe schlechthin und bezogen auf Verdienste: "großer Frieden», "großes Vermächtnis», "großes Werk» und "großes Erbe», sowie durch die Erwähnung der 49 Orden, unter denen sich zwei «Großkreuze» befanden ${ }^{14}$.

Worin diese Größe besteht, wird ebenfalls ausgespart, so daß der Begriff ausgehöhlt und die Leere mit spielerischen Collagen gefüllt wird. «Wer ist groß?» lautet die wiederholte Frage (Kiesingers), und die dazwischengeschnittenen Vorschläge lauten: «Churchill, de Gasperi, Schumann, Bismarck, John Foster Dulles, Pius» und enden in Gerstenmeiers halblautem Einwurf «niemals»! ${ }^{15}$.

Eine zweite Reihe dieser Art nennt den «Hl. Paulus, Haba-kuk, Gott im Himmel, ein Greis, der Gerechte, ein deutscher Staatsmann, ein geachteter Partner - in der Kathedrale von Reims». Hier wird die Frage schließlich ausgespart, und die Aufzählung schlägt um in eine Paraphrasierung der mystifizierten Person. Das Ratespiel wird ein drittes Mal aufgenommen mit dem Vorschlag «Die Tholeyer Sängerknaben», und durch die Provinzialität wird Größe nun bis zum Lächerlichen relativiert.

Provinzialität tritt auch im regional gefärbten Deutsch der Redner auf sowie in der Evozierung verschiedener Anekdoten aus dem Leben Adenauers und der Erinnerung an Zitate, die zu geflügelten Worten wurden, zum Beispiel der «kölsche Klüngel».

Doch gerade die Entstehung geflügelter Worte ist ein Indiz für Prominenz, deren Popularität und letzendlich Macht, die sich sprachlich manifestiert. Öffentliche Sprache ist wie das Denken jedes Einzelnen durchdrungen von den sprachlichen 
Gewohnheiten der mächtigen Autorität und wird zum Indiz für Identifikation. Obwohl gleichzeitig Autorität auch ein wenig lächerlich erscheint, wird sie in dieser Form der Herabziehung auf ein alltägliches und triviales Maß eher noch populärer statt eigentlich in Frage gestellt. Dies wird sie erst durch die Veränderung des Kontextes, der das Funktionieren solcher Elemente im Hörspiel-Experiment deutlich macht.

Weitere Häufungen sind Superlative zum Ausdruck von Macht: der Dom wurde als «größte Kirche der Welt geplant», er ist eine «gewaltige Basilika», «die größte Basilika nördlich der Alpen», und die Trauerfeierlichkeiten sind die "größten der Bundesrepublik» ${ }^{16}$.

Der Bürger bleibt dabei anonyme Masse: «Tausende» und «Millionen» ${ }^{17}$, während die Ehrengäste namentlich genannt werden und in eine Reihe von weiteren Elementen einer Oben-Unten-Dichotomie eingegliedert werden: «hochverehrte Trauerversammlung», «hohe Spitzenvertreter», «hohe Gäste», der «hohe Dom», der "hohe Chor», der «Hochaltar» sowie der Blick des Reporters am seitlichen Westturm des Doms empor und der Blick der dort stationierten Ordnungskräfte herab auf die Menge.

Dieser Blick wird verlängert bis zum Himmel als Anspielung auf das Karfreitagsgeschehen und damit als weiterer Hinweis auf die Vergöttlichung der Staatsmacht. Zahlreiche Anspielungen ergänzen dies: so die Bemerkung, daß der Mensch «von oben stamme», die Analogie zu Moses und daß «seine (Adenauers, U.E.) Stimme zu uns spricht».

Göttlichkeit der Staatsmacht wird hier beschrieben, und zwar geht es hier um eine durch Zeremonie in Medien hergestellte Überhöhung. Als quasi göttliche Macht entzieht sie sich der Legitimation und Kontrolle durch die Öffentlichkeit.

öffentliche Sprache wird als Pose und bewußte Irreführung bloßgestellt, indem Harig den Kontext seiner Zitate verändert: «Er war eher auf Skepsis als auf rasche Gutgläubigkeit gestimmt $»^{18}$. Das gleiche gilt für die vielgerühmte Einfachheit: «Das Wort der Einfachheit [...] paßt nur auf die Sprache, die er in der Öffentlichkeit führte» ${ }^{19}$.

Während der Bürger sich dem metaphysischen Idol der Staatsmacht unterwirft, nimmt sich dieses ihm gegenüber alle Freiheiten heraus, so lautet das implizite Fazit eines solchen Stückes und zwar «bis an die Grenzen des Erlaubten $»^{20}$.

57 Hier entfällt endgültig der Humor, der stellenweise das Stück aufheitert. Es geht nämlich nicht darum, Macht durch Lachen zu entschärfen, sondern die Täuschung zu erkennen, der sich der Medienrezipient bereitwillig hingibt, d.h. das Stück muß als eine Aufforderung verstanden werden, den «Glauben» an die staatliche Macht und bzw. die Vertreter dieser Macht aufzugeben.

Durch die Anonymisierung zeigt Harig generelles Funktionieren staatlicher Macht auf, wobei auch die Bedrohung deutlich wird, die von einer vermeintlich väterlichen charismatischen Persönlichkeit wie Adenauer ausgehen kann, wenn sie ihre Macht mißbraucht, und zwar ohne daß Harig auf andere historische Beispiele rekurriert. 


\section{NOTES}

1. - Hörspiele entstehen auch als Produktionen freier, privater Studios. Der Vertrieb erfolgt über Verlage oder unmittelbar als Kassette oder Schallplatte. Eine Zusammenarbeit des öffentlichrechtlichen Rundfunks mit privaten Studios kommt auch bei Hörspielproduktionen mitunter vor und ist dispositionsbedingt.

2. - Elisabeth Noelle-Neumann, Winfried Schulz, Jürgen Wilke (Hrsg.), Massenkommunikation. Frankfurt: Fischer 1989, S. 75.

3. - Helmut Heißenbüttel, Hörspielpraxis und Hörspielhypothese, in: Akzente, Zeitschrift für Literatur, hrsg. v. Walter Höherer und Hans Bender. 16. Jg. 1969, S. 23-29.

4. - Hellmut Geissner, Spiel mit Hörer, in: Akzente 16. Jg. 1969, S. 32.

5. - Bernd Peter Arnold, Hanns Verres, Radio. München TR Verlagsunion 1989, S. 109. Die Autoren unterscheiden im soziologischen Sinn 7 Realitäten: 1. objektive Realität, 2. Realität der biologischen Art, 3. die subjektive Realität eines Kulturkreises, 4. die subjektive Realität einer sozialen Gruppe, 5. die subjektive Realität des Individuums, 6. vorgetäuschte Realität, 7. Medienrealität.

6. - Louis MacNeice, Christopher Columbus, a radio play. London 1944, Introduction, cit. cf. Christian W. Thomsen, Das englische Hörspiel, in: ders. u. Irmela Schneider, Grundzüge der Geschichte des europäischen Hörspiels, Darmstadt: Wissenschaftliche Buchgesellschaft 1985, S. 20.

7. - Saarländischer Rundfunk 1989 in der Realisation von Heidrun Nass.

8. - Friederike Mayröcker, Raymond Federman, Walter Jens, Wüstensturm. Bayerischer Rundfunk in einer Koproduktion mit dem Saarländischen Rundfunk 1990.

9. - Saarländischer Rundfunk 1969, Koproduktion mit dem Westdeutschen Rundfunk.

10. - Heinrich Vormweg: Dokumente und Collagen, in: Neue Hefte - Essays, S. 159.

11. - Adenauers früherer Staatssekretär Globke war für die Organisation der Zeremonien verantwortlich. Nach Hans-Peter Schwarz (Adenauer: Der Staatsmann 1952-1967, Stuttgart: DVA 1991, S. 180 f.) ist sie in Deutschland nur mit dem Begräbnis Wilhelms I. vergleichbar, sowohl was die Zurschaustellung staatlicher, militärischer und kirchlicher Macht betrifft, als auch die Anteilnahme der Bevölkerung.

12. - Bodo Würffei, Das deutsche Hörspiel, Stuttgart: Metzler 1978, S. 175f. Zur sprach- und ideologiekritischen Funktion des experimentellen Hörspiels cf. Hellmut Geissner, Spiel mit Hörer, in: Akzente, 16. Jg. 1969, S. 41.

13. - SR-Funkmanuskript S. 4, 26, 30, 31.

14. - Ebd. S. 11, 9, 14, 25, 4.

15. - Ebd. S. 11.

16. - Ebd. S. 5 und 11.

17. - Ebd. S. 5, 9, 10, 21.

18. - Ebd. S. 28 und 29.

19. - Ebd. S. 14 und 15.

20. - Ebd. S. 19 und 22. 


\section{RÉSUMÉS}

Das Hörspiel unterscheidet sich von den anderen Sendeformen des Rundfunks durch die Freiheit in der Wahl seiner Form, so daß es nicht wie diese bestimmten Hörererwartungen ausgesetzt ist. Bei der Wahl eines aktuellen Themas kann es sich jedoch auch einer Sendeform bedienen, die primär der aktuellen Berichterstattung dient. Dadurch erzeugt es ästhetische und kritische Wirkungen, die anhand von drei Beispielen illustriert werden: «Gestörtes Leben» von W. Schiffer, «Wüstensturm» von F. Mayröcker, R. Federman und W. Jens sowie «Staatsbegräbni» von L. Harig. Diese Stücke enthalten nicht nur einen Inhalt, der unter bestimmten Aspekten präsentiert ist, sondern schließen gleichzeitig eine kritische Reflexion des Mediums und seiner Rezeption ein.

La pièce radiophonique peut librement choisir sa forme et diffère en cela des autres genres de ce média dont les genres bien définis correspondent chez l'auditeur à une attente spécifique. La pièce qui aborde un sujet d'actualité peut utiliser également la forme d'une émission dont la fonction primordiale est de présenter l'actualité. Elle engendre ainsi des effets esthétiques et critiques illustrés par trois exemples: «Gestörtes Leben» de W. Schiffer, «Wüstensturm» de F. Mayröcker, R. Federman et W. Jens et «Staatsbegräbnis» de L. Harig. Les pièces montrent non seulement l'intrigue sous un ou plusieurs aspects critiques et émotionnels, mais la forme implique une réflexion et une critique du média même, ainsi que des habitudes de réception de l'auditeur. 